\title{
The expansion of Hall-Littlewood functions in the dual Grothendieck polynomial basis
}

\author{
Jason Bandlow ${ }^{1}$ and Jennifer Morse ${ }^{2}$ \\ ${ }^{1}$ University of Pennsylvania, Philadelphia, PA, USA \\ ${ }^{2}$ Drexel University, Philadelphia, PA, USA
}

\begin{abstract}
A combinatorial expansion of the Hall-Littlewood functions into the Schur basis of symmetric functions was first given by Lascoux and Schützenberger, with their discovery of the charge statistic. A combinatorial expansion of stable Grassmannian Grothendieck polynomials into monomials was first given by Buch, using set-valued tableaux. The dual basis of the stable Grothendieck polynomials was given a combinatorial expansion into monomials by Lam and Pylyavskyy using reverse plane partitions. We generalize charge to set-valued tableaux and use all of these combinatorial ideas to give a nice expansion of Hall-Littlewood polynomials into the dual Grothendieck basis.

Résumé. En associant une charge à un tableau, une formule combinatoire donnant le développement des polynômes de Hall-Littlewood en termes des fonctions de Schur a été obtenue par Lascoux et Schützenberger. Une formule combinatoire donnant le développement des polynômes de Grothendieck Grassmanniens stables en termes des fonctions monomiales a quant à elle été obtenue par Buch à l'aide de tableaux à valeurs sur des ensembles. Finalement, une formule faisant intervenir des partitions planaires inverses a été obtenue par Lam et Pylyavskyy pour donner le développement de la base duale aux polynômes de Grothendieck stables en termes de monômes. Nous généralisons le concept de charge aux tableaux à valeurs sur des ensembles et, en nous servant de toutes ces notions combinatoires, nous obtenons une formule élégante donnant le développement des polynômes de Hall-Littlewood en termes de la base de Grothendieck duale.
\end{abstract}

Keywords: symmetric functions, Hall-Littlewood polynomials, Grothendieck polynomials, charge statistic

\section{Introduction}

The Hall-Littlewood functions are symmetric functions with a wealth of applications. In various forms, they interpolate between the complete homogeneous and Schur basis of symmetric functions, provide a polynomial realization of the Hall algebra, and have several interpretations as characters of representations. Lascoux and Schützenberger gave an expansion of the Hall-Littlewood functions into Schur functions, in terms of a statistic on tableaux called charge [LS78]. We write this expansion as

$$
H_{\mu}[X ; t]=\sum_{\lambda} \sum_{T \in S S T(\lambda, \mu)} t^{c h(T)} s_{\lambda}
$$

where $S S T(\lambda, \mu)$ is the set of all semi-standard tableaux of shape $\lambda$ and evaluation $\mu$. From this expansion, the following properties of the Hall-Littlewood function are more or less immediate:

1365-8050 (c) 2010 Discrete Mathematics and Theoretical Computer Science (DMTCS), Nancy, France 
- the coefficient of $s_{\lambda}$ is a polynomial in $t$ with non-negative integer coefficients,

- specializing $t=1$ gives $H_{\mu}[X ; 1]=h_{\mu}$, the complete homogeneous symmetric function, and

- specializing $t=0$ gives $H_{\mu}[X ; 0]=s_{\mu}$.

See [Mac95] for more details about Hall-Littlewood functions.

The Grothendieck polynomials were introduced by Lascoux and Schützenberger [LS83] as power series representatives for the $K$-theory classes of the structure sheaves of Schubert varieties. The stable Grothendieck polynomials introduced by Fomin and Kirillov [FK94] are the stable limit of these as the number of variables approaches infinity. These functions, written $G_{\lambda}$, are non-homogeneous symmetric functions, which cannot be written as a finite sum of Schur functions. The function $G_{\lambda}$ is equal to $s_{\lambda}$ in its lowest degree homogeneous component, and (in terms of Schur functions or monomials) is sign-alternating by degree in the higher homogeneous components. Buch gave an expansion of the stable Grothendieck polynomials into monomial symmetric functions by introducing set-valued tableaux [Buc02]. We write this expansion as

$$
G_{\lambda}=\sum_{\mu}(-1)^{|\mu|-|\lambda|} k_{\lambda, \mu} m_{\mu}
$$

where $k_{\lambda, \mu}$ denotes the number of set-valued tableaux of shape $\lambda$ and evaluation $\mu$.

Lam and Pylyavskyy studied the dual basis to the stable Grothendieck polynomials under the Hall inner product [LP07]. They expanded these into monomials using a special evaluation of reverse plane partitions. We denote the dual basis to $\left\{G_{\lambda}\right\}$ by $\left\{g_{\lambda}\right\}$. These are Schur positive, non-homogeneous symmetric functions, with $g_{\lambda}$ equal to $s_{\lambda}$ in the top degree. We note that equation 2 and a simple fact about dual bases immediately implies

$$
h_{\mu}=\sum_{\lambda}(-1)^{|\mu|-|\lambda|} k_{\lambda, \mu} g_{\lambda} .
$$

In this work, we give a generalization of the charge statistic to set-valued tableaux. In particular, we define the reading word of a set-valued tableau, and then define the charge to be the charge of the reading word. We then prove the common generalization of equations 1 and 3 .

$$
H_{\mu}[X ; t]=\sum_{\lambda}(-1)^{|\mu|-|\lambda|} \sum_{T \in S V T(\lambda, \mu)} t^{c h(T)} g_{\lambda}
$$

where $\operatorname{SVT}(\lambda, \mu)$ denotes the set of set-valued tableaux of shape $\lambda$ and evaluation $\mu$. We find it remarkable that such a nice formula exists, as we are unaware of any direct connection between the HallLittlewood functions and $K$-theory.

\section{Definitions and notation}

\subsection{Symmetric function basics}

We begin by setting our notation with some standard definitions. An introduction to symmetric functions can be found in [Mac95] or [Sta99]. 
Definition 1 The Young diagram of a partition $\lambda=\left(\lambda_{1}, \lambda_{2}, \cdots, \lambda_{k}\right)$ is a left- and bottom-justified array of $1 \times 1$ square cells in the first quadrant of the coordinate plane, with $\lambda_{i}$ cells in the $i^{\text {th }}$ row from the bottom.

Example 1 The Young diagram of the partition $(3,2)$ is \begin{tabular}{l}
\hline \\
$\square$
\end{tabular} .

Definition 2 A semi-standard tableau of shape $\lambda$ is a filling of the cells in the Young diagram of $\lambda$ with positive integers, such that the entries

- are weakly increasing while moving rightward across any row, and

- are strictly increasing while moving up any column.

Example 2 A semi-standard tableau of shape $(3,2)$ is \begin{tabular}{|l|l|l|}
\hline 2 & 3 & \\
\hline 1 & 1 & 2 \\
\hline
\end{tabular} .

The evaluation of a semi-standard tableau is the sequence $\left(\alpha_{i}\right)_{i \in \mathbb{N}}$ where $\alpha_{i}$ is the number of cells containing $i$. The evaluation of the tableau in example 2 is $(2,2,1)$ (trailing 0 's have been omitted, as is customary). We use the notation $S S T(\lambda)$ to mean the set of all semi-standard tableaux of shape $\lambda$, and $S S T(\lambda, \mu)$ to mean the set of all semi-standard tableaux of shape $\lambda$ and evaluation $\mu$.

The Schur functions have many definitions, one of which is in terms of semi-standard tableaux.

Definition 3 The Schur function $s_{\lambda}$ is defined by

$$
s_{\lambda}=\sum_{T \in S S T(\lambda)} \mathbf{x}^{e v(T)} .
$$

The notation $\mathbf{x}^{e v(T)}$ means $x_{1}^{\alpha_{1}} x_{2}^{\alpha_{2}} \cdots$, where $\left(\alpha_{1}, \alpha_{2}, \cdots\right)$ is the evaluation of $T$. The Schur functions are elements of $\mathbb{C}\left[\left[x_{1}, x_{2}, \cdots\right]\right]$, the power series ring in infinitely many variables, and are well known to be a basis for the symmetric functions (i.e., those elements of $\mathbb{C}\left[\left[x_{1}, x_{2}, \cdots\right]\right]$ which are invariant under any permutation of their indices).

Example 3 The Schurfunction $s_{(2,1)}$ can be written as

$$
s_{(2,1)}=x_{1}^{2} x_{2}+x_{1} x_{2}^{2}+2 x_{1} x_{2} x_{3}+\cdots
$$

corresponding to the tableaux

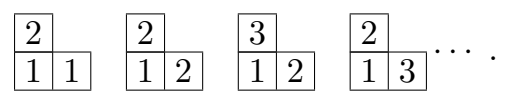

Another basis for the symmetric functions is the monomial symmetric functions.

Definition 4 The monomial symmetric function $m_{\lambda}$ is defined by

$$
m_{\lambda}=\sum_{\alpha} \mathbf{x}^{\alpha}
$$

where the sum is over all sequences $\alpha$ which are a rearrangement of the parts of $\lambda$. (Here $\lambda$ is thought of as having finitely many non-zero parts, followed by infinitely many 0 parts.) 
Example 4 The monomial symmetric function $m_{(2,1)}$ can be written as

$$
m_{(2,1)}=x_{1}^{2} x_{2}+x_{1} x_{2}^{2}+x_{1}^{2} x_{3}+x_{1} x_{3}^{2}+x_{2}^{2} x_{3}+x_{2} x_{3}^{2}+\ldots
$$

The Kostka numbers give the change of basis matrix between the Schur and monomial symmetric functions. For two partitions $\lambda$, $\mu$, we define the number $K_{\lambda, \mu}$ to be number of semi-standard tableaux of shape $\lambda$ and evaluation $\mu$. From the previous definitions, one can see that a consequence of the symmetry of the Schur functions is that

$$
s_{\lambda}=\sum_{\mu} K_{\lambda, \mu} m_{\mu} .
$$

There is a standard inner product on the vector space of symmetric functions (known as the Hall inner product), defined by setting

$$
\left\langle s_{\lambda}, s_{\mu}\right\rangle=\left\{\begin{array}{lc}
1 & \text { if } \lambda=\mu \\
0 & \text { otherwise }
\end{array}\right.
$$

The following proposition is a basic, but very useful, fact of linear algebra.

Proposition 1 If $\left(\left\{f_{\lambda}\right\},\left\{f_{\lambda}^{*}\right\}\right)$ and $\left(\left\{g_{\lambda}\right\},\left\{g_{\lambda}^{*}\right\}\right)$ are two pairs of dual bases for an inner-product space, and

$$
f_{\lambda}=\sum_{\mu} M_{\lambda, \mu} g_{\mu}
$$

then

$$
g_{\mu}^{*}=\sum_{\lambda} M_{\lambda, \mu} f_{\lambda}^{*}
$$

We define the set of homogeneous symmetric functions, $\left\{h_{\lambda}\right\}$, to be the dual-basis to the monomial symmetric functions. An immediate consequence of proposition 1 is that

$$
h_{\mu}=\sum_{\lambda} K_{\lambda, \mu} s_{\lambda}
$$

\subsection{Hall-Littlewood symmetric functions}

The Hall-Littlewood functions belong to the space $\mathbb{C}[t]\left[\left[x_{1}, x_{2}, \ldots\right]\right]$ of formal power series in infinitely many variables with coefficients in the polynomial ring $\mathbb{C}[t]$. There are multiple (unequal, but related) definitions of these functions in the literature. The version we concern ourselves with here are most commonly written as $H_{\lambda}$ or $Q_{\lambda}^{\prime}$. In [LS78], Lascoux and Schützenberger found a purely combinatorial presentation of these functions. The key notion is a statistic on semi-standard tableaux known as charge. Before defining charge, we need the notion of the reading word of a tableau.

Definition 5 The reading word of a tableau $T$, which we denote by $w(T)$, is the sequence $\left(w_{1}, w_{2}, \ldots, w_{n}\right)$ obtained by listing the elements of $T$ starting from the top-left corner, and reading across each row and then continuing down the rows.

Example 5 We have $w\left(\begin{array}{|l|l|l}\hline 2 & 3 & \\ \hline 1 & 1 & 2\end{array}\right)=(2,3,1,1,2)$. 
We will first define the charge of a word, and then define the charge of a tableau to be the charge of its reading word. For our purposes, it will be sufficient to define charge only on words whose evaluation is a partition. While this can be extended to all semi-standard tableau, this requires a substantially more complicated definition. We begin by defining the charge of a permutation; this is a word with evaluation $(1,1, \ldots, 1)$. If $w$ is a permutation of length $n$, then the charge of $w$ is given by $\sum_{i=1}^{n} c_{i}(w)$ where $c_{1}(w)=0$ and $c_{i}(w)$ is defined recursively as

$$
c_{i}(w)=c_{i-1}(w)+\chi(i \text { appears to the right of } i-1 \text { in } w) .
$$

Here we use the notation that when $P$ is a proposition, $\chi(P)$ is equal to 1 if $P$ is true and 0 if $P$ is false.

Example 6 A straightforward computation shows that

$$
\operatorname{ch}(3,5,1,4,2)=0+1+1+2+2=6 .
$$

We will now describe the decomposition of a word with partition content into charge subwords, each of which are permutations. The charge of a word will then be defined as the sum of the charge of its charge subwords. To find the first charge subword $w^{(1)}$ of a word $w$, we begin at the right of $w$ (i.e., at the last element of $w$ ) and move leftward through the word, marking the first 1 that we see. After marking a 1, we continue to travel to the left, now marking the first 2 that we see. If we reach the beginning of the word, we loop back to the end. We continue in this manner, marking successively larger elements, until we have marked the largest letter in $w$, at which point we stop. The subword of $w$ consisting of the marked elements (with relative order preserved) is the first charge subword. We then remove the marked elements from $w$ to obtain a word $w^{\prime}$. The process then continues iteratively, with the second charge subword being the first charge subword of $w^{\prime}$, and so on.

Example 7 We illustrate the first charge subword of $w=(5,2,3,4,4,1,1,1,2,2,3)$ by labeling the relevant elements in bold: $(\mathbf{5}, \mathbf{2}, 3,4, \mathbf{4}, 1,1, \mathbf{1}, 2,2, \mathbf{3})$. If we remove the bold letters, and bold the second charge subword, we obtain $(\mathbf{3}, \mathbf{4}, 1, \mathbf{1}, 2, \mathbf{2})$. It is now easy to see that the third and final charge subword is $(\mathbf{1}, \mathbf{2})$. Thus we have the following computation of the charge of $w$ :

$$
\begin{aligned}
\operatorname{ch}(w) & =\operatorname{ch}(5,2,4,1,3)+\operatorname{ch}(3,4,1,2)+\operatorname{ch}(1,2) \\
& =(0+0+1+1+1)+(0+1+1+2)+(0+1) \\
& =8
\end{aligned}
$$

We can now define the Hall-Littlewood polynomials.

Definition 6 The Hall-Littlewood polynomial $H_{\lambda}[X ; t]$ is defined by

$$
H_{\mu}[X ; t]=\sum_{\lambda} \sum_{T \in S S T(\lambda, \mu)} t^{c h(T)} s_{\lambda}
$$

Note the similarity of this definition and equation (6). In particular, if we set $t=1$ in definition 6, we obtain equation (6) exactly. 


\subsection{Grothendieck polynomials}

To define the Grothendieck polynomials, we need first need a definition of set-valued tableaux, due to Buch [Buc02].

Definition 7 A set-valued tableau of shape $\lambda$ is a filling of the cells in the Young diagram of $\lambda$ with sets of positive integers, such that

- the maximum element in any cell is weakly smaller than the minimum element of the cell to its right, and

- the maximum element in any cell is strictly smaller than the minimum entry of the cell above it.

Another way to think about this definition is that if we select a single element from each cell (in any possible way) we will always end up with a semi-standard tableau.

\section{Example 8}

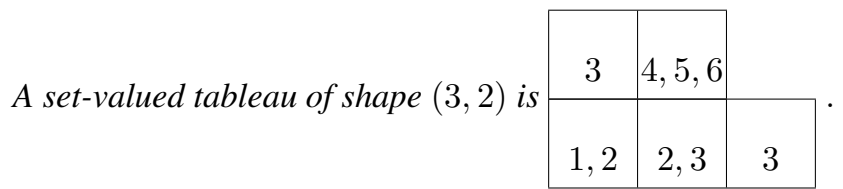

We have omitted the set braces, ' $\{$ ' and ' $\}$ ', here and throughout for clarity.

The evaluation of a set-valued tableaux $S$ is the composition $\alpha=\left(\alpha_{i}\right)_{i \geq 1}$ where $\alpha_{i}$ is the total number of times $i$ appears in $S$. For example, the evaluation of the tableau in example 8 is $(1,2,3,1,1,1)$. The collection of all set-valued tableaux of shape $\lambda$ will be denoted $S V T(\lambda)$ and the collection of all setvalued tableaux of shape $\lambda$ and evaluation $\alpha$ will be denoted $S V T(\lambda, \alpha)$. We write $k_{\lambda, \mu}$ for the number of set-valued tableaux of shape $\lambda$ and evaluation $\mu$.

We will use set-valued tableaux to define the Grothendieck polynomials.

Definition 8 We define the polynomials $G_{\lambda}(X)$ by

$$
G_{\lambda}=\sum_{\mu}(-1)^{|\mu|-|\lambda|} k_{\lambda, \mu} m_{\mu}
$$

We note that when $|\mu|=|\lambda|$, we must have one element in every cell; hence $G_{\lambda}$ is equal to $s_{\lambda}$ plus higher degree terms. Since the $G_{\lambda}$ are known to be symmetric functions, they must therefore form a basis.

Applying proposition 1 to this definition gives

$$
h_{\mu}=\sum_{\mu}(-1)^{|\mu|-|\lambda|} k_{\lambda, \mu} g_{\lambda}
$$

where the $\left\{g_{\lambda}\right\}$ are the dual basis to the $\left\{G_{\lambda}\right\}$.

The dual Grothendieck polynomials $g_{\lambda}$ were studied by Lam and Pylyavskyy [LP07]. They gave an expansion of the $g_{\lambda}$ into monomials via reverse plane partitions.

Definition $9 A$ reverse plane partition of shape $\lambda$ is a filling of the cells in the Young diagram of $\lambda$ with positive integers, such that the entries are weakly increasing in rows and columns. 
Example 9 A reverse plane partition of shape $(3,2)$ is \begin{tabular}{l|l|l|l|}
\hline 1 & 2 & \\
\hline 1 & 1 & 2 \\
\end{tabular}.

Following Lam and Pylyavskyy (and differing from some other conventions) we define the evaluation of a reverse plane partition $P$ to be the composition $\alpha=\left(\alpha_{i}\right)_{i \geq 1}$ where $\alpha_{i}$ is the total number of columns in which $i$ appears. For example, the evaluation of the reverse plane partition in example 9 is $(2,2)$. The collection of all reverse plane partitions of shape $\lambda$ will be denoted $R P P(\lambda)$ and the collection of all reverse plane partitions of shape $\lambda$ and evaluation $\alpha$ will be denoted $\operatorname{RPP}(\lambda, \alpha)$.

Theorem 1 (Lam-Pylyavskyy) The polynomials $g_{\lambda}$ have the expansion

$$
g_{\lambda}=\sum_{T \in R P P(\lambda)} \mathbf{x}^{e v(P)}
$$

We note that when $|\mu|=|\lambda|$, the entries must be strictly increasing up columns; hence $g_{\lambda}$ is equal to $s_{\lambda}$ plus lower degree terms.

\section{Main result}

Before we can state our result, we must define the charge of a set-valued tableau. This is accomplished by defining the reading word of a set-valued tableau.

Definition 10 The reading word of a set-valued tableau $T$, which we denote by $w(T)$, is the sequence $\left(w_{1}, w_{2}, \ldots, w_{n}\right)$ obtained by listing the elements of $T$ starting from the top-left corner, and reading each row according to the following procedure and then continuing down the rows. In each row, we first ignore the smallest element of each cell, and read the remaining elements from right to left, and from largest to smallest within each cell. Then we read the smallest element of each cell from left to right, and proceed to the next row.

Example 10 The reading word of

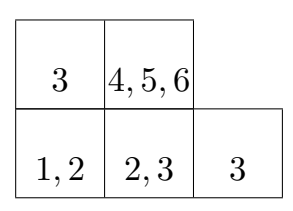

is $(6,5,3,4,3,2,1,2,3)$.

We now define the charge of set-valued tableau to be the charge of its reading word. We may now state our main theorem.

Theorem 2 We have the following expansion of Hall-Littlewood functions into dual Grothendieck functions:

$$
H_{\mu}[X ; t]=\sum_{\lambda}(-1)^{|\mu|-|\lambda|} \sum_{S \in S V T(\lambda, \mu)} t^{c h(S)} g_{\lambda}
$$


As this is an extended abstract, we provide only a sketch of the proof. Expanding the $s_{\lambda}$ on the right hand side of equation 1 gives

$$
\begin{aligned}
H_{\mu}[X ; t] & =\sum_{\lambda} \sum_{T \in S S Y T(\lambda, \mu)} t^{\operatorname{ch}(T)} s_{\lambda} \\
& =\sum_{\lambda} \sum_{T \in S S Y T(\lambda, \mu)} t^{\operatorname{ch}(T)} \sum_{Q \in S S Y T(\lambda)} \mathbf{x}^{e v(Q)} .
\end{aligned}
$$

If we expand the $g_{\lambda}$ in (9) according to theorem 11, we obtain

$$
H_{\mu}[X ; t]=\sum_{\lambda}(-1)^{|\mu|-|\lambda|} \sum_{S \in S V T(\lambda, \mu)} t^{c h(S)} \sum_{R \in R P P(\lambda)} \mathbf{x}^{e v(R)}
$$

Now we define $\mathfrak{S}_{\mu}$ to be the set of pairs $(S, R)$ where $S$ is a set-valued tableau of evaluation $\mu$ and $R$ is a reverse plane-partition of the same shape as $S$. We define the sign of such a pair to be $(-1)^{|\mu|-|\lambda|}$ (where $\lambda$ is the mutual shape of $S$ and $R$ ) and the weight of such a pair to be $t^{\operatorname{ch}(S)} \mathbf{x}^{e v(R)}$. Comparing 10 and [12, we see that we can complete the proof by finding a sign-reversing, weight-preserving involution on $\mathfrak{S}_{\mu}$ whose fixed points are pairs $(S, R)$ where both $S$ and $R$ are semi-standard in the usual sense. We describe this involution below.

\subsection{Definition of the involution}

Given a pair $(S, R)$ we wish to construct a pair $\iota(S, R)=\left(S^{\prime}, R^{\prime}\right)$ of opposite sign and equal weight. We start from the top of both tableaux and work our way down until we find the first row where at least one of the following conditions hold:

1. A cell in $S$ contains more than one element.

2. A cell in $R$ contains the same element as the cell immediately above it.

If no such row exists, the pair is a fixed point. Otherwise, we define $\operatorname{row}(S, R)$ to be this row. If only condition (1) holds in $\operatorname{row}(S, R)$, we will perform an operation we call expansion to define $\left(S^{\prime}, R^{\prime}\right)$. Alternatively, if only condition 2 holds in $\operatorname{row}(S, R)$, we perform an operation called contraction to define $\left(S^{\prime}, R^{\prime}\right)$. If both conditions hold, we will either expand or contract; the method for determining which will be described following the description of the operations.

We first describe expansion, beginning with the construction of $S^{\prime}$. Let $i=\operatorname{row}(S, R)$, and define $x(S, R)$ to be the largest element in row $i$ of $S$ which is contained in a multi-element cell (henceforth, multicell). Let $\widehat{S}$ be the semi-standard tableau consisting of the rows of $S$ which are strictly above row $i$. We form $S^{\prime}$ from $S$ by removing $x(S, R)$ from the multi-cell in row $i$ which contains it, and replacing $\widehat{S}$ with the Schensted insertion $x(S, R) \rightarrow \widehat{S}$. Let $c$ be the cell $S^{\prime} \backslash S$. We form $R^{\prime}$ from $R$ by placing an empty marker in the cell $c$ and sliding this marker to the south-west using jeu-de-taquin. When the marker reaches row $i$ of $R$, we replace it with the entry in the cell directly above it. This is $R^{\prime}$.

We now describe contraction, beginning with the construction of $R^{\prime}$. Again, we let $i=\operatorname{row}(S, R)$. We begin by replacing with an empty marker the rightmost cell in row $i+1$ of $R$ which has the same value as the cell immediately below it. Using reverse jeu-de-taquin, slide this marker up and to the right until it 
exits the diagram. This is $R^{\prime}$. Let $c$ be the cell $R \backslash R^{\prime}$. To construct $S^{\prime}$, we again let $\widehat{S}$ be the semi-standard tableau consisting of the rows of $S$ which are strictly above row $i$. Then we perform reverse Schensted insertion on the element in cell $c$ to get a semi-standard tableaux $\widehat{S}^{\prime}$ and an element $y(S, R)$. Finally, we place $y(S, R)$ inside an existing cell in row $i$; there will be a unique cell in this row into which we can place $y(S, R)$ so that the result is a valid set-valued row. $S^{\prime}$ is then defined by placing $\widehat{S}^{\prime}$ on top of this modified row $i$, on top of the remaining lower rows of $S$.

If both conditions 1 and 2 hold in row $\operatorname{row}(S, R)$, we must decide whether to perform expansion or contraction. We expand if $x(S, R) \geq y(S, R)$ and contract otherwise. This justifies the claim that, in the contraction case, there is a unique cell in row $i$ into which we can place the element $y(S, R)$; there will never be an element $\geq y(S, R)$ in a multi-cell in row $i$. Thus $y(S, R)$ can placed (and must be placed) in the rightmost cell such that all of its elements are $\leq y(S, R)$. Such a cell must exist since in $S, y(S, R)$ was an element of row $i+1$.

As this is an extended abstract, we omit the proof that this is a weight-preserving sign-reversing involution. However, we give a simple example below.

Example 11 The involution $\iota$ exchanges the two pairs below:

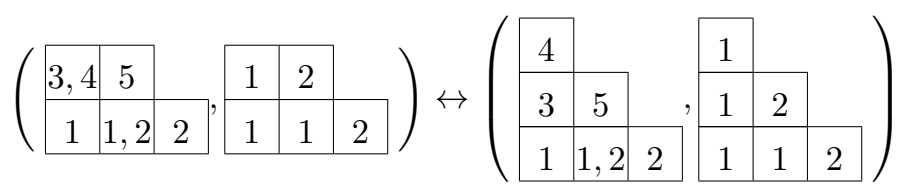

These pairs have opposite sign, and common weight $t^{2} x_{1}^{2} x_{2}^{2}$.

\section{References}

[Buc02] Anders Skovsted Buch. A Littlewood-Richardson rule for the $K$-theory of Grassmannians. Acta Math., 189(1):37-78, 2002.

[FK94] Sergey Fomin and Anatol N. Kirillov. Grothendieck polynomials and the Yang-Baxter equation. In Formal power series and algebraic combinatorics/Séries formelles et combinatoire algébrique, pages 183-189. DIMACS, Piscataway, NJ, 1994.

[LP07] Thomas Lam and Pavlo Pylyavskyy. Combinatorial Hopf algebras and $K$-homology of Grassmannians. Int. Math. Res. Not. IMRN, (24):Art. ID rnm125, 48, 2007.

[LS78] Alain Lascoux and Marcel-Paul Schützenberger. Sur une conjecture de H. O. Foulkes. $C$. $R$. Acad. Sci. Paris Sér. A-B, 286(7):A323-A324, 1978.

[LS83] Alain Lascoux and Marcel-Paul Schützenberger. Symmetry and flag manifolds. In Invariant theory (Montecatini, 1982), volume 996 of Lecture Notes in Math., pages 118-144. Springer, Berlin, 1983.

[Mac95] Ian G. Macdonald. Symmetric Functions and Hall Polynomials. The Clarendon Press, Oxford University Press, New York, second edition, 1995. With contributions by A. Zelevinsky.

[Sta99] Richard P. Stanley. Enumerative Combinatorics, volume 2. Cambridge University Press, Cambridge, United Kingdom, 1999. 
